\title{
Insight into Protein Conformation and Subcharging by DMSO from Native Ion Mobility Mass Spectrometry
}

\author{
Daniel Shiu-Hin Chan, Dijana Matak-Vinković, Anthony G. Coyne, and Chris Abell*
}

\begin{abstract}
Electrospray ionization-mass spectrometry (ESI-MS) interfaced with ion-mobility (IM) spectrometry has enabled the study of protein structure and interactions under native-like conditions. In biological assays, dimethyl sulfoxide (DMSO) is often included as a co-solvent to dissolve organic molecules. While low levels of DMSO are known to reduce the charge of protein ions generated by ESI, the exact mechanism by which this occurs has been debated. In this study, we describe the first application of IM-MS to study the effect of DMSO subcharging on native protein conformation. We find that at low concentrations, DMSO induces modest (1-2\%), but repeatable, reductions in protein collision-cross sections (CCSs) of four different protein complexes, avidin, concanavalin A, alcohol dehydrogenase, and pyruvate kinase, as measured by travelingwave (TW) IM-MS. Individual protein charge states also experienced compaction in size, suggesting that this effect could not be attributed to the shift of charge state distribution by DMSO alone.
\end{abstract}

Electrospray ionization-mass spectroscopy (ESI-MS) has emerged as a versatile technique to probe protein structure and function. ${ }^{[1-3]}$ Additionally, the interfacing of ESI-MS with ionmobility (IM) spectrometry, termed ion-mobility mass spectrometry (IM-MS), provides an additional dimension of resolution whereby ions are separated by their collision crosssection (CCS) as they traverse a chamber of neutral gas particles under the influence of an electric field..$^{[4-7]}$

Biological screening systems will often contain significant quantities (up to $10 \%$ ) of dimethyl sulfoxide (DMSO), particularly where high concentrations of weakly-binding ligands such as fragments are used. ${ }^{[8-9]}$ Interestingly, low DMSO concentrations $(<10 \%)$ decrease the average charge of protein ions generated by ESI, whereas higher concentrations ( $>10 \%$ ) of DMSO lead to an increase in average charge and also induce a broadening of charge state distributions. ${ }^{[10-14]}$ However, the mechanism by which low concentrations of DMSO decrease charge is still under debate. ${ }^{[13,15]}$ Williams and co-workers have attributed the charge-reducing effects of DMSO to a "global compaction" of protein structure, as supported by both circular dichroism (CD) and hydrogen-deuterium exchange (HDX) experiments in solution, ${ }^{[11]}$ while contrasting data from Tjernberg and coworkers appear to show destabilization, degradation and aggregation of proteins at DMSO concentrations as low as $0.5 \% .{ }^{[10]}$ Other additives that have been reported to reduce charge in ESI include DMF, ${ }^{[15]}$ imidazole,${ }^{[16]}$ and various solvent vapors. ${ }^{[17]}$

This work aims clarify whether the supposed compaction of protein structure at low concentrations of DMSO is manifested as a change in CCS that can be detected by traveling-wave ionmobility mass spectrometry (TWIM-MS) for four different proteins. To our knowledge, this is the first time that native IMMS has been applied to study the effect of DMSO on protein conformation. These results provide further insight into the charge-reducing mechanism of DMSO and may also help to reconcile previous observations regarding this phenomenon.

Avidin (egg white), concanavalin A (Canavalia ensiformis), alcohol dehydrogenase (Saccharomyces cerevisiae) and pyruvate kinase (rabbit heart) were prepared at $20 \mu \mathrm{M}$ concentration in $\mathrm{NH}_{4} \mathrm{OAc}$ buffer $(200 \mathrm{mM}, \mathrm{pH}$ 7.0) containing 0 to $20 \%$ DMSO. These four tetrameric proteins were chosen due to their facile electrospray behavior as well as CCS values that have been previously determined by drift-tube (DT) IM-MS technology. ${ }^{[18]}$ Protein solutions were subjected to nanoelectrospray ionization (nESI) ${ }^{[19]}$ using a hybrid quadrupole timeof-flight (qTOF) SYNAPT HDMS (Waters) instrument equipped with a traveling wave ion-mobility (TWIM) device.

Native mass spectra of avidin (Figure 1a), concanavalin A (Figure S1a), alcohol dehydrogenase (Figure S2a) and pyruvate kinase (Figure S3a) in the absence of DMSO indicated that their quaternary structures were well-preserved, with the tetramer presenting as the predominant species in all cases. For concanavalin A, a significant amount of the dimeric protein and a small quantity of the monomeric protein were also observed, consistent with previous reports, ${ }^{[12,}{ }^{20]}$ whereas some nonspecific dimerization of tetramers was observed in the case of avidin.

As the concentration of DMSO was increased from 0 to $20 \%$, the charge state distributions of the four protein complexes were observed to shift to higher $\mathrm{m} / \mathrm{z}$ values (lower charge) at low DMSO concentrations, followed by a reversal of the trend towards lower $\mathrm{m} / \mathrm{z}$ values (higher charge) at higher DMSO concentrations (Figures 1a, S1a-S3a), as is consistent with previous reports. ${ }^{[10-11,13]}$ For example, the abundance-weighted average charge of avidin decreased monotonically from $15.5+$ to $14.1+$ as the DMSO concentration was increased from $0 \%$ to $4 \%$, followed by an increase to $17.3+$ as the DMSO concentration was increased to $20 \%$ (Figure $1 \mathrm{~b}$ ).

$\left.{ }^{*}{ }^{\star}\right] \quad$ D. S.-H. Chan, Dr. D. Matak-Vinković, Dr. A. G. Coyne, Prof. C Abell Department of Chemistry University of Cambridge Lensfield Road, Cambridge CB2 1EW (United Kingdom) E-mail:ca26@cam.ac.uk

Supporting information for this article is given via a link at the end of the document. 
a)

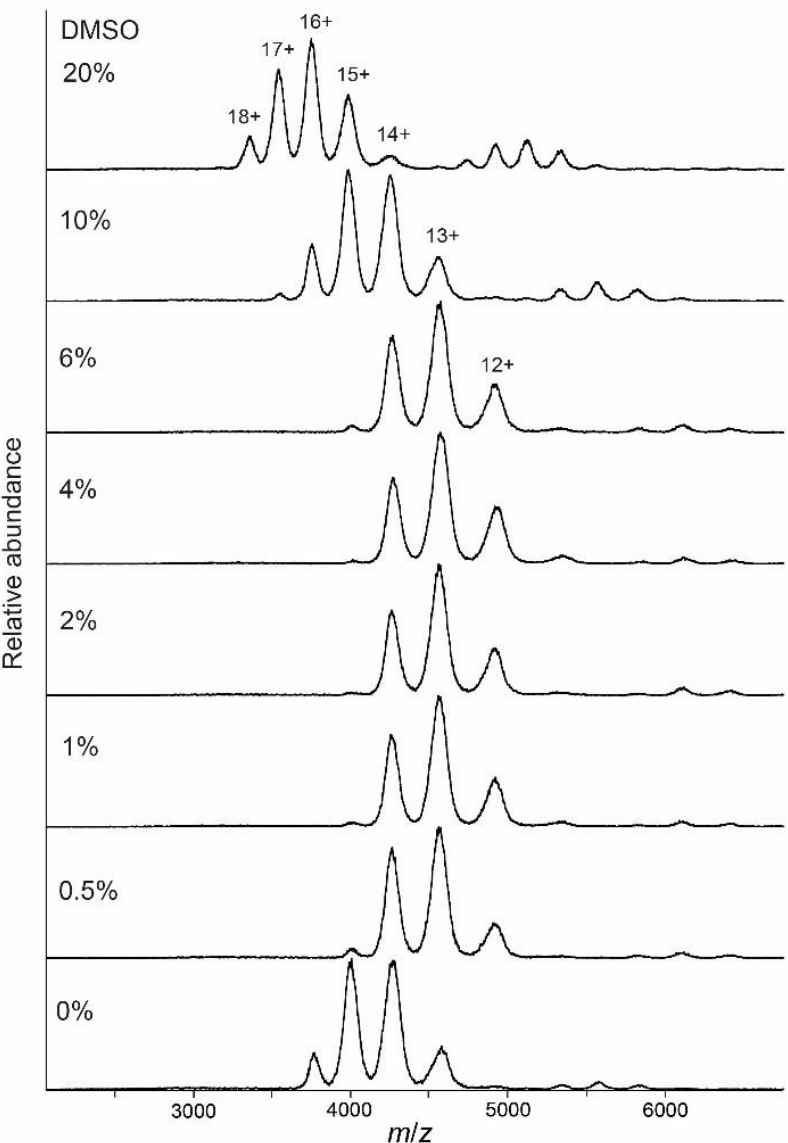

b)
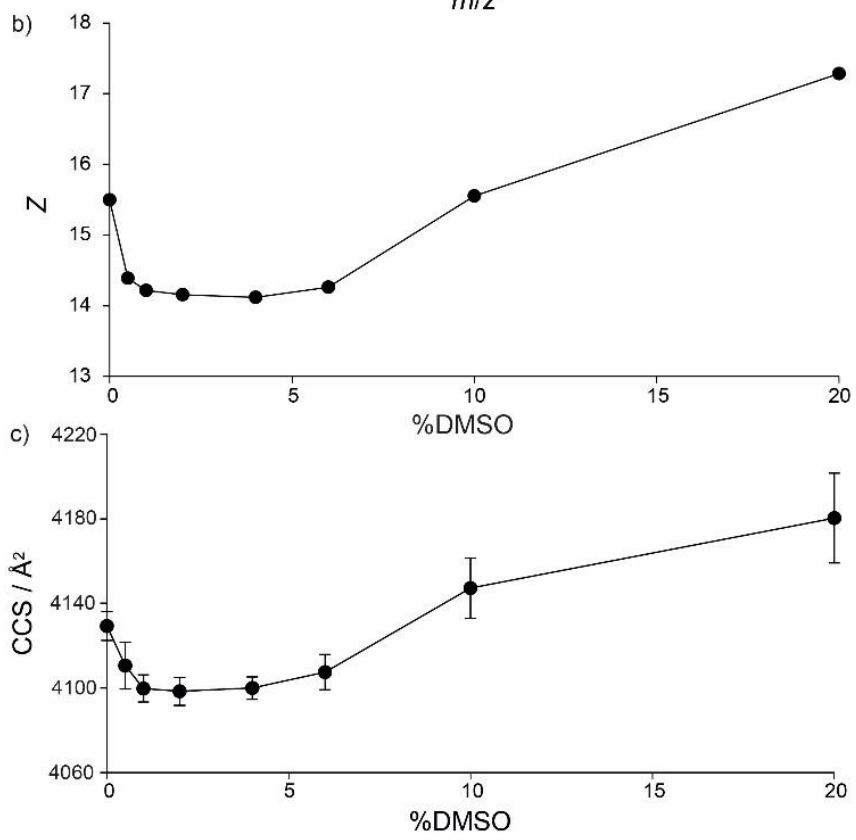

Figure 1. a) $\mathrm{nESI}-\mathrm{MS}$ spectra of avidin $(20 \mu \mathrm{M})$ in $\mathrm{NH}_{4} \mathrm{OAc}(200 \mathrm{mM}, \mathrm{pH} 7.0)$ containing $0,0.5,1,2,4,6,10$ or $20 \%$ DMSO. b) Weighted-average charge of avidin tetramers as a function of DMSO concentration. c) Weighted-average CCS of avidin tetramers as a function of DMSO concentration. Error bars represent the standard deviation of replicate results.

The minimum average charge for the four proteins occurred at 2 or $4 \%$ concentration of DMSO (Figure 1b, S1b-
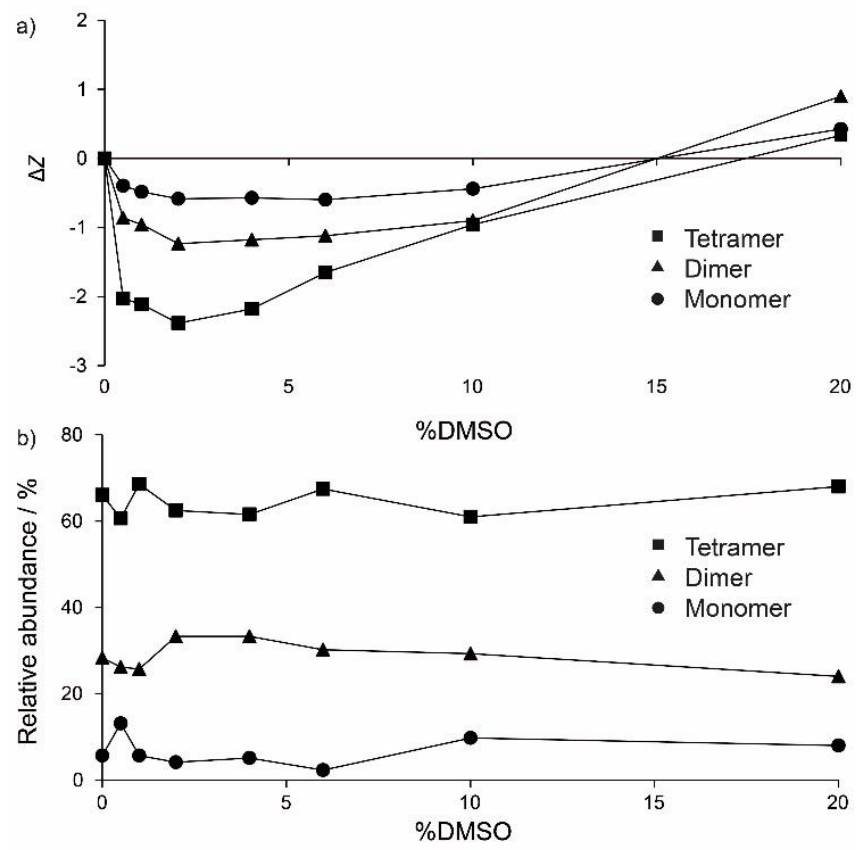

Figure 2. a) Relative charge reduction of concanavalin A tetramers, dimers and monomers as a function of DMSO concentration. b) Relative abundance of concanavalin A tetramers, dimers and monomers as a function of DMSO concentration.

S3b). Furthermore, concanavalin A dimers and monomers showed similar charging behavior in response to DMSO concentration as compared to the tetramers (Figure 2a). For all of the protein complexes, significant charge distribution broadening was not apparent until DMSO concentrations reached high values $(\geq 10 \%)$. At $20 \% \mathrm{DMSO}$, the average charge of the proteins became greater than their initial values in the absence of DMSO, suggesting the onset of global protein unfolding, which could account for the increase in the number of occupied charge states at higher DMSO concentrations, ${ }^{[21]}$ as well as the mild increase in the amount of dimerized avidin tetramers (Figure 1a). It should be noted that because of the higher boiling point of DMSO compared to water, ESI droplets become enriched in DMSO (estimated to be 3 to 5 -fold in Williams et $a{ }^{[11]}$ ) during the desolvation process, and hence the effective DMSO concentration experienced by the gaseous protein ions is higher than in solution.

A previous report found that $2.5 \%$ DMSO induced significant dissociation of the bacterial NAD+ synthase from dimeric into monomeric form, possibly via weakening of the hydrophobic effect. ${ }^{[22]}$ In comparison, no dissociation of any of the four protein tetramers was observed by native MS at any DMSO concentration. Moreover, the tetramer-dimer-monomer equilibrium for concanavalin A was not significantly perturbed by the addition of up to $20 \%$ of DMSO (Figure 2b). This also contrasts with observations from supercharging experiments, in which the addition of as little as $0.5 \%$ of $m$-nitrobenzyl alcohol ( $m$-NBA) resulted in significant dissociation of concanavalin A dimers into monomers. ${ }^{[12]}$ This suggests that the chargereducing effects of DMSO protect against, or at least do not promote, subunit dissociation in ESI in this case.

CCS values for the protein complexes at various DMSO concentrations were obtained in $\mathrm{N}_{2}$ and calibrated with $\mathrm{CCS}^{\mathrm{N} 2}$ 


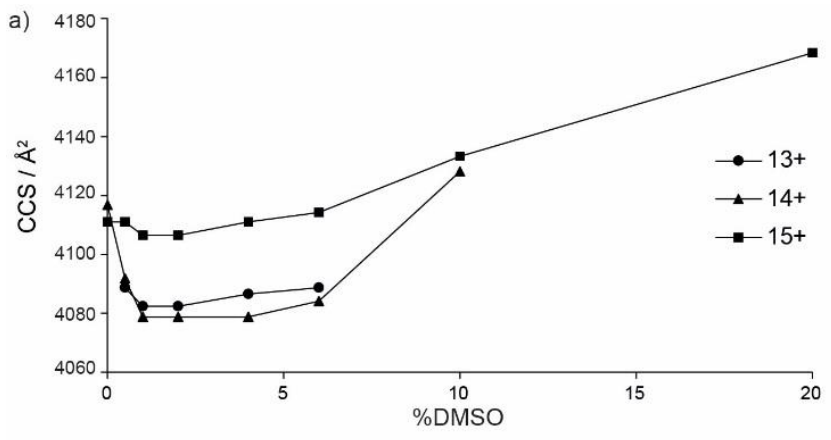

b)
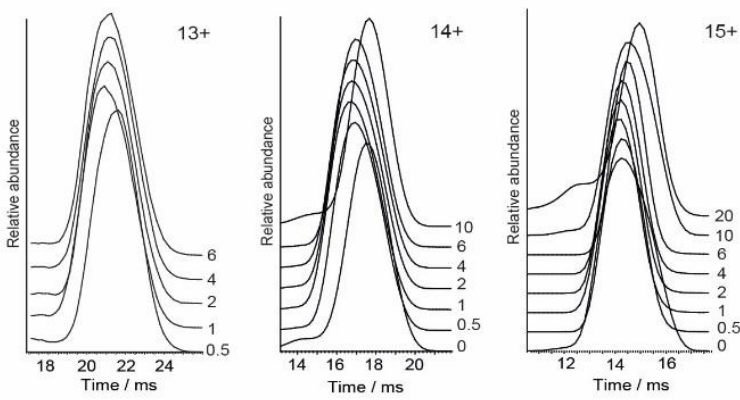

Figure 3. a) CCS of $13+, 14+$ and $15+$ charge states of avidin tetramers as a function of DMSO concentration. b) Arrival time distributions of the 13+, 14+ and $15+$ charge states of avidin at wave height $7 \mathrm{~V}$ across DMSO concentrations. DMSO concentrations are indicated at the side of the plot.

reference values for the proteins under DMSO-free conditions following the procedure of Ruotolo and Robinson et al. ${ }^{[23]}$ Experiments were performed at four different wave heights $(7,8$, 9 and $10 \mathrm{~V}$ ) to rule out the effect of electric field on gaseous ion separation. Intriguingly, the abundance-weighted average CCS of the four proteins underwent a decrease in value at low DMSO concentrations followed by an increase at higher DMSO levels, paralleling the trend observed for average charge (Figure 1c, S1c-S3c). For example, the average CCS of the avidin tetramer decreased from $4130 \pm 10 \AA^{2}$ at $0 \%$ DMSO (DT CCS $^{\mathrm{N} 2}=4150$ $\left.\AA^{2}\right)^{[18]}$ to $4090 \pm 10 \AA^{2}$ at $2 \%$ DMSO, followed by an increase to $4200 \pm 30 \AA^{2}$ at $20 \%$ DMSO (Figure 1c) (see the Supporting Information for error analysis). While these differences are small (ca. 1-2\%), the overall trends were repeatable from independently-performed experiments.

The minimum CCS values for the four proteins occurred at 1 or $2 \%$ DMSO (Figures 1c, S1c-S3c), which are similar to the DMSO concentrations at which minimum charge was reached. At $20 \%$ DMSO, the average CCS of the proteins became higher than their initial values under DMSO-free conditions, which is consistent with preliminary unfolding and the onset of protein supercharging at this DMSO concentration. These data would therefore support the idea of global protein compaction at low DMSO levels, ${ }^{[11]}$ followed by the onset of protein unraveling at higher concentrations.

Higher charge states of a protein generally have larger CCSs than lower charge states of the same protein, which has been attributed to increased Coulombic repulsion leading to unfolding in the gas phase, or alternatively, to the transfer of less compact proteins from solution that presumably provide greater surface area for charge development. ${ }^{[2]}$ Is the apparent compaction of protein structure at low DMSO concentrations a direct consequence of the charge-reducing capability of DMSO, allowing protein ions to access lower charge states that have decreased internal repulsion, or is protein compaction an $a b$ initio effect by DMSO in solution that in turn promotes charge reduction in the gas phase?

To investigate this issue, the relationship between the CCSs for single charge states and DMSO concentration was analyzed. If the observed compaction of protein structure was solely due to the ability of DMSO to shift the protein charge state distribution to ions of lower charge, then the CCSs of individual charge states should not be appreciably different from each other regardless of DMSO concentration. Interestingly, for several of the charge states that are common to a range of DMSO concentrations, their CCSs exhibited the characteristic bimodal behavior in response to DMSO concentration (Figures $3 a, 54 a-c)$ as for average charge and average CCS values. This behavior was also evident from consideration of the arrival time distributions (ATDs) for individual charge states (Figures 3b, S5a-h), which shift to lower drift times at lower DMSO concentrations (indicating protein compaction), followed by a shift to larger drift times at higher DMSO concentrations. These observations argue against the hypothesis that the decreased Coulombic repulsion afforded by DMSO-induced charge reduction is the sole factor driving the overall compaction of the protein.

We also observe that in the subcharging regime, lower charge states do not necessarily exhibit smaller CCSs than higher charge states at a given DMSO concentration. This is similar to observations from the Robinson group showing that serum amyloid $\mathrm{P}$ component (SAP) and aldehyde dehydrogenase complexes subjected to charge reduction by triethylammonium acetate (TEA) do not show significant changes in CCS. ${ }^{[25]}$ Moreover, DTIM-MS measurements of native-like, non-supercharged proteins suggest that the positive correlation between charge and CCS is present only for smaller proteins, whereas for larger proteins or protein complexes, this relationship is not apparent. ${ }^{[18,26]}$ This further suggests that internal repulsion should not have a significant effect on the CCSs of the different charge states of the proteins in this study.

The observations above suggest that protein compaction by DMSO may instead be occurring in solution, which has been previously documented for lysozyme by the groups of Voets ${ }^{[27]}$ and Williams ${ }^{[11]}$ on the basis of $\mathrm{CD}$, small-angle neutron scattering, Rayleigh scattering and HDX-MS results. This compaction of protein structure in solution could then be transferred into the gas-phase for it to be detected by TWIM-MS, as we have shown in this report. However, while this could potentially account for the charge reduction of protein ions at low DMSO concentrations, ${ }^{[11]}$ we consider that this is unlikely to be the only operative mechanism, as the degree of CCS reduction (ca. 1-2\%) was small in comparison to the magnitude of charge reduction (ca. 10\%).

Quantitatively, Marijnissen and co-workers have suggested based on theoretical considerations that the charge of a progeny droplet generated during electrospray is proportional to droplet surface area raised to the power of $3 / 4 \cdot{ }^{[28]}$ Assuming that the entirety of this charge is transferred to the encapsulated protein when the droplet evaporates, this relationship should hold true for protein ions generated by ESI. ${ }^{[29]}$ By correlating the average charge of proteins observed in ESI-MS with their predicted 
surface areas from crystal structures, Kaltashov and co-workers determined that the charge-surface area relationship followed a power function of $0.69 \pm 0.02 .^{[29]}$ In our study, plotting a log-log graph of average charge against average CCS for the four proteins under DMSO-free conditions revealed an excellent linear fit $\left(R^{2}=0.997\right)$, with a power constant of 0.78 (Figure S6a) However, log-log plots for the individual complexes under varying DMSO concentrations showed both worse fits $\left(R^{2}=0.82\right.$ to 0.97 ) as well as much higher power constants of 4.5 to 10.6 (Figure S6b), suggesting that the DMSO-induced change in CCS, and by extension surface area, is itself insufficient to account for the entirety of the change in charge.

Instead, other chemical or physical mechanisms, such as gas-phase proton transfer ${ }^{[13]}$ or dissociative cooling, ${ }^{[15-16]}$ may account for the observed subcharging of protein ions at low DMSO concentrations. Additionally, we do not preclude the possibility that the observed differences in CCS might be due to other factors; for example, gaseous ions might, depending on the DMSO concentration, vary in their internal energy as a result of different desolvation, leading to small changes in drift time. In the specific case of ring-like protein assemblies, initial compaction can also be achieved through collision activation. ${ }^{[30]}$

The precise mechanisms governing the relationships between electrospray conditions, protein charge and CCS values are still not completely understood. In particular, a consistent framework that accounts for the charge-reducing effects of DMSO in ESI has not yet been fully developed. Against this backdrop, this study has provided evidence for the first time of a modest, but repeatable, decrease of protein size at low DMSO concentrations. Our data also suggest that this compaction is unlikely to be solely attributable to the shift of charge state distribution by DMSO, as different charge states of the same protein were also individually compacted by DMSO, and also because internal Coulombic repulsion is unlikely to be a dominant factor for these systems. Instead, our results lead us to speculate that the previously reported compaction of protein structure by DMSO in solution can be adequately maintained upon transfer to the gas-phase, allowing for this phenomenon to be detected by TWIM-MS. However, the observed CCS reduction is relatively small and is thus unlikely to account for the entirety of charge reduction by DMSO, suggesting that alternative subcharging mechanisms are operative. Finally, and in contrast to previous studies with other proteins, we observe no evidence of gross protein denaturation, degradation or aggregation (except for mild dimerization of avidin) of proteins under study at $4{ }^{\circ} \mathrm{C}$ over the timescale of days even at $20 \%$ DMSO. This suggests that the deleterious effects of DMSO on protein structure and stability are highly protein-dependent, and further reinforces the notion that the DMSO concentration for each biological assay has to be carefully optimized in order to ensure that the results are not compromised by unintended effects of DMSO on protein binding or activity.

Experimental methods, supplementary spectra and error analysis can be found in the Supporting Information. Additional data related to this publication can be accessed at the University of Cambridge data repository

(http://dx.doi.org/10.17863/CAM.5953).

\section{Acknowledgements}

D.S.-H. Chan acknowledges the support of the Croucher Foundation and the Cambridge Commonwealth, European and International Trust for receipt of a Croucher Cambridge International Scholarship.

Keywords: DMSO $\cdot$ electrospray $•$ ion mobility $•$ mass spectrometry $\cdot$ subcharging

[1] K. Rajabi, A. E. Ashcroft, S. E. Radford, Methods 2015, 89, 13-21

[2] G. R. Hilton, J. L. P. Benesch, J. R. Soc. Interface 2012, 9, 801 816 .

[3] 816. 12275.

[4] F. Lanucara, S. W. Holman, C. J. Gray, C. E. Eyers, Nat. Chem 2014, 6, 281-294.

[5] J. L. P. Benesch, B. T. Ruotolo, Curr. Opin. Struct. Biol. 2011, 21, 641-649.

[6] M. Sharon, A. Horovitz, Curr. Opin. Struct. Biol. 2015, 34, 7-16.

[7] K. J. Pacholarz, R. A. Garlish, R. J. Taylor, P. E. Barran, Chem. Soc. Rev. 2012, 41, 4335-4355.

[8] R. Macarrón, R. P. Hertzberg, Mol. Biotechnol. 2010, 47, 270-285

[9] E. H. Mashalidis, P. Śledź, S. Lang, C. Abell, Nat. Protocols 2013 8, 2309-2324.

[10] A. Tjernberg, N. Markova, W. J. Griffiths, D. Hallén, J. Biomol. Screen. 2006, 11, 131-137.

[11] H. J. Sterling, J. S. Prell, C. A. Cassou, E. R. Williams, J. Am. Soc Mass Spectrom. 2011, 22, 1178-1186.

[12] H. J. Sterling, A. F. Kintzer, G. K. Feld, C. A. Cassou, B. A. Krantz E. R. Williams, J. Am. Soc. Mass Spectrom. 2012, 23, 191-200.

[13] R. R. O. Loo, R. Lakshmanan, J. A. Loo, J. Am. Soc. Mass Spectrom. 2014, 25, 1675-1693.

[14] E. R. Dickinson, E. Jurneczko, J. Nicholson, T. R. Hupp, J. Zawacka-Pankau, G. Selivanova, P. E. Barran, Front. Mol. Biosci. 2015, 2, 39.

[15] M. Landreh, G. Alvelius, J. Johansson, H. Jörnvall, Anal. Chem. 2014, 86, 4135-4139.

[16] D. Bagal, E. N. Kitova, L. Liu, A. El-Hawiet, P. D. Schnier, J. S. Klassen, Anal. Chem. 2009, 81, 7801-7806. J. T. S. Hopper, K. Sokratous, N. J. Oldham, Anal. Biochem. 2012 421, 788-790.

M. F. Bush, Z. Hall, K. Giles, J. Hoyes, C. V. Robinson, B. T. Ruotolo, Anal. Chem. 2010, 82, 9557-9565.

H. Hernandez, C. V. Robinson, Nat. Protocols 2007, 2, 715-726. D. F. Senear, D. C. Teller, Biochemistry 1981, 20, 3076-3083. I. A. Kaltashov, R. R. Abzalimov, J. Am. Soc. Mass Spectrom. 2008, 19, 1239-1246.

22] Z. W. Yang, S. W. Tendian, W. M. Carson, W. J. Brouillette, L. J. Delucas, C. G. Brouillette, Protein Sci. 2004, 13, 830-841.

B. T. Ruotolo, J. L. P. Benesch, A. M. Sandercock, S.-J. Hyung, C. V. Robinson, Nat. Protocols 2008, 3, 1139-1152.

[24] E. Jurneczko, P. E. Barran, Analyst 2011, 136, 20-28.

[25] Z. Hall, C. V. Robinson, J. Am. Soc. Mass Spectrom. 2012, 23 1161-1168.

[26] R. Salbo, M. F. Bush, H. Naver, I. Campuzano, C. V. Robinson, I. Pettersson, T. J. D. Jørgensen, K. F. Haselmann, Rapid Commun. Mass Spectrom. 2012, 26, 1181-1193.

[27] I. K. Voets, W. A. Cruz, C. Moitzi, P. Lindner, E. P. G. Arêas, P. Schurtenberger, J. Phys. Chem. B 2010, 114, 11875-11883. R. P. A. Hartman, D. J. Brunner, D. M. A. Camelot, J. C. M. Marijnissen, B. Scarlett, J. Aerosol Sci. 2000, 31, 65-95.
I. A. Kaltashov, A. Mohimen, Anal. Chem. 2005, 77, 5370-5379.

[29] I. A. Kaltashov, A. Mohimen, Anal. Chem. 2005, 77, 5370-5379. Chem. Soc. 2012, 134, 3429-3438. 
Entry for the Table of Contents (Please choose one layout)

Layout 1:

\section{COMMUNICATION}

This study provides evidence for the first time of a modest, but repeatable, decrease of protein size at low DMSO concentrations. Different charge states of the same protein were also individually compacted by DMSO, suggesting that the previously reported compaction of protein structure by DMSO in solution can be adequately maintained upon transfer to the gas-phase, allowing for this phenomenon to be detected by TWIMMS

Layout 2:

\section{COMMUNICATION}

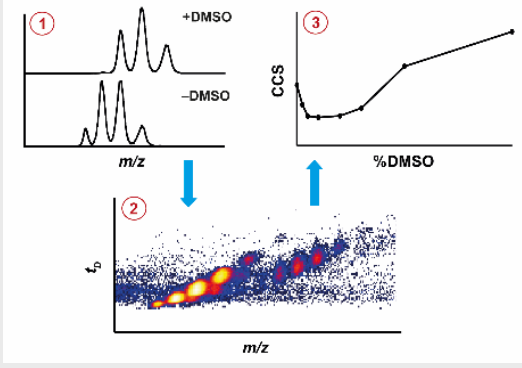

Daniel S.-H. Chan, Dijana MatakVinković, Anthony G. Coyne, Chris Abell*

Page No. - Page No.

Insight into Protein Conformation and Subcharging by DMSO from Native Ion Mobility Mass Spectrometry
((Insert TOC Graphic here))

Text for Table of Contents
Author(s), Corresponding Author(s)*

Page No. - Page No.

Title 
Additional Author information for the electronic version of the article.

$\begin{array}{ll}\text { Author: } & \text { ORCID identifier } \\ \text { Author: } & \text { ORCID identifier }\end{array}$

Author: ORCID identifier 